\title{
Chiral Pharmaceutical Intermediaries Obtained by Reduction of 2-Halo-1-(4-substituted phenyl)-ethanones Mediated by Geotrichum candidum CCT 1205 and Rhodotorula glutinis CCT 2182
}

\author{
Lucídio C. Fardelone, J. Augusto R. Rodrigues, and Paulo J. S. Moran \\ Institute of Chemistry, University of Campinas, CP 6154, 13084-971 Campinas, SP, Brazil \\ Correspondence should be addressed to Paulo J. S. Moran, moran@iqm.unicamp.br
}

Received 14 January 2011; Accepted 10 March 2011

Academic Editor: Robert F. H. Dekker

Copyright () 2011 Lucídio C. Fardelone et al. This is an open access article distributed under the Creative Commons Attribution License, which permits unrestricted use, distribution, and reproduction in any medium, provided the original work is properly cited.

\begin{abstract}
Enantioselective reductions of $p-\mathrm{R}_{1}-\mathrm{C}_{6} \mathrm{H}_{4} \mathrm{C}(\mathrm{O}) \mathrm{CH}_{2} \mathrm{R}_{2}\left(\mathrm{R}_{1}=\mathrm{Cl}, \mathrm{Br}, \mathrm{CH}_{3}, \mathrm{OCH}_{3}, \mathrm{NO}_{2}\right.$ and $\left.\mathrm{R}_{2}=\mathrm{Br}, \mathrm{Cl}\right)$ mediated by Geotrichum candidum CCT 1205 and Rhodotorula glutinis CCT 2182 afforded the corresponding halohydrins with complementary $R$ and $S$ configurations, respectively, in excellent yield and enantiomeric excesses. The obtained $(R)$ - or $(S)$-halohydrins are important building blocks in chemical and pharmaceutical industries.
\end{abstract}

\section{Introduction}

Chiral halohydrins are important and valuable intermediates in the synthesis of fine chemicals and pharmaceuticals as optically active 1,2-aminoalcohols. The halohydrin $(R)$-1-aryl2-haloethanol may be used for the preparation of $(R)$-1-aryl2 -aminoethanols that are used as $\alpha$ - and $\beta$-adrenergic drugs.

An interesting chemoenzymatic synthetic route to obtain optically active 1 -aryl-2-ethanolamines is from the enantioselective reduction of the correspondent $\alpha$-haloacetophenones giving halohydrins that are transformed into an epoxy that reacts with the appropriate amine (Scheme 1) $[1,2]$.

An enormous potential of the use of microorganisms and enzymes for the transformation of synthetic chemicals with high chemo-, regio-, and enantioselectivity has been increasing in the pharmaceutical industry [3]. The dehydrogenases in the form of whole cells for the production of chiral styrene oxides have been used on a pilot-plant scale [4]. Therefore, a large number of papers have appeared reporting the enantiomeric reduction of $\alpha$-bromoacetophenone [5$10]$ and $\alpha$-chloroacetophenone $[4,6,7,11-17]$ by whole cells of microorganism and also by isolated enzyme [18] giving halohydrins in high enantiomeric excesses (ee).
There are few examples of biocatalytic reduction of $\alpha$-haloacetophenone having suitable substituted group attached to the aromatic ring for enantioselective preparation of some target 1-aryl-2-ethanolamines $[2,19]$. It is known that some examples of biocatalytic reductions of $\alpha$-haloacetophenone that have substituted groups like 3-chloro [20, 21], 4-nitro $[10,22]$, and 3,4-methylenedioxy [23-25] were mediated by a number of microorganisms. Also, isolated enzymes have been used to reduce $\alpha$-haloacetophenone having various kinds of substituted groups $[26,27]$.

The performances of Rhodotorula glutinis CCT 2182 and Geotrichum candidum CCT 1205 in bioreduction of $\alpha$ haloacetophenone have been calling our attention due to the efficiency and complementary enantioselectivity of these microorganisms giving the corresponding $(R)$ - and $(S)$ halohydrins in high ee, respectively [8]. Also, those microorganisms show the same efficiency in the reduction of $\alpha$ azido-para-substituted acetophenones [28]. In this work, we use those two microorganisms for reduction of $\alpha$-bromoand $\alpha$-chloroacetophenones having para-substituted groups to produce separately both enantiomers of halohydrins that can be used as chiral building blocks for preparations of the corresponding 1,2-aminoalcohols. 


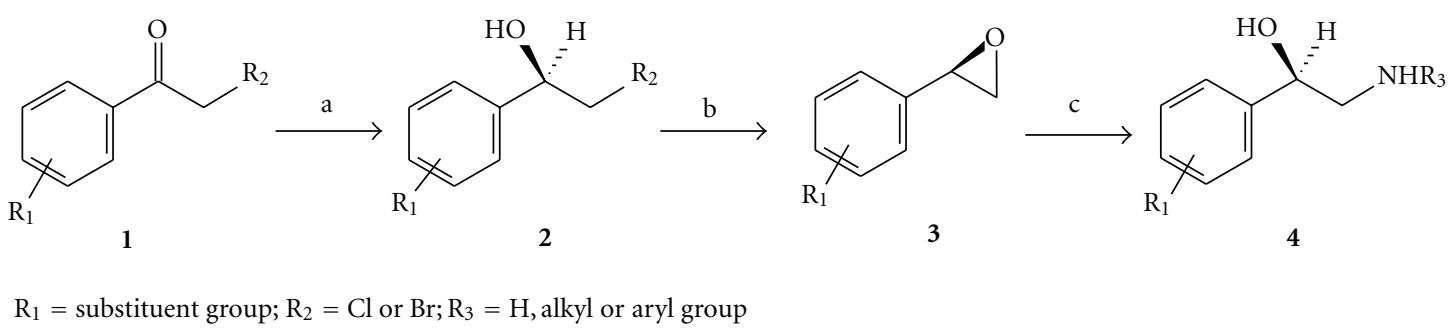

Scheme 1: (a) reduction using chiral catalytic reagent or biocatalytic process; (b) base; (c) amine.

\section{Materials and Methods}

IR spectra were recorded on a Bomem MB Series spectrometer. ${ }^{1} \mathrm{H}$ and ${ }^{13} \mathrm{C}$ NMR spectra were recorded on a Varian Gemini 300 spectrometer in $\mathrm{CDCl}_{3}$. Gas chromatographic analyses were performed using a Shimadzu GC/MS Class 5000, with helium as carrier gas. The fused silica capillary columns used were either a Supelco Simplicity ITM $(30 \mathrm{~m} \times 0.25 \mathrm{~mm} \times 0.25 \mu \mathrm{m})$ and a chiral GC-column CHIRASILDEX $(30 \mathrm{~m} \times 0.25 \mathrm{~mm} \times 0.25 \mu \mathrm{m})$. Optical rotation was measured with a J-720, VRDM306 JASCO, $589.3 \mathrm{~nm}\left(25^{\circ} \mathrm{C}\right)$ spectropolarimeter. The melting points were obtained in MQAPF-301-MicroQuímica equipment.

The 2-bromo-1-(4-substituted phenyl)-1-ethanones 1a-e were obtained with brominating 4-substituted acetophenones in $\mathrm{CH}_{2} \mathrm{Cl}_{2}$ at $0^{\circ} \mathrm{C}$, and 2-chloro-1-(4-substituted phenyl)-1-ethanones $\mathbf{1 f}-\mathbf{j}$ were prepared applying the Wyman and Kaufman methodology [29] by chlorination of corresponding 4-substituted acetophenones with sulfuryl chloride in $\mathrm{CH}_{2} \mathrm{Cl}_{2}$ at $0^{\circ} \mathrm{C}$. All other reagents and solvents were reagent grade.

The racemic 2-halo-1-(4-substituted phenyl)-ethanols $2 \mathbf{a}-\mathbf{j}$, used as reference for the determination of ee in a GC provided with a chiral column, were obtained by reacting the corresponding $\mathbf{1} \mathbf{a}-\mathbf{j}$ with $\mathrm{NaBH}_{4}$ in water/methanol at rt. All other solvents and reagents were reagent grade.

2.1. Growth Conditions for Microorganisms Culture. The microorganisms Geotrichum candidum CCT 1205 (isolated from industrial waste water treatment-Preston, United Kington) and Rhodotorula glutinis CCT 2182 (isolated from Psidium guajava-Atlantic Rainforest, Brazil) were stored at "André Tosello" Research Foundation (Campinas, Brazil) [30]. G. candidum was cultivated in $400 \mathrm{~mL}$ of nutrient broth $1(10 \mathrm{~g} / \mathrm{L}$ malt extract, $5 \mathrm{~g} / \mathrm{L}$ peptone, $10 \mathrm{~g} / \mathrm{L}$ glucose, $3.12 \mathrm{~g} / \mathrm{L}$ $\mathrm{K}_{2} \mathrm{HPO}_{4}$, and $\left.11.18 \mathrm{~g} / \mathrm{L} \mathrm{KH}_{2} \mathrm{PO}_{4}\right)$ at $28^{\circ} \mathrm{C}$, and $R$. glutinis was cultivated in $400 \mathrm{~mL}$ of nutrient broth $2(3 \mathrm{~g} / \mathrm{L}$ Yeast extract, $3 \mathrm{~g} / \mathrm{L}$ malt extract, $5 \mathrm{~g} / \mathrm{L}$ peptone, and $10 \mathrm{~g} / \mathrm{L}$ glucose) at $30^{\circ} \mathrm{C}$. Both yeasts were incubated for 2 days on an orbital shaker $(200 \mathrm{rpm})$ before use. All materials and medium were sterilized in an autoclave at $121^{\circ} \mathrm{C}$ before use and the yeasts were manipulated in a laminar flow cabinet.

2.2. General Procedure for Bioreduction of 2-Halo-1-(4-substituted phenyl)-ethanones. The yeasts were incubated for two days $(400 \mathrm{~mL}$ nutrient broth in Erlenmeyer of $1 \mathrm{~L})$. After that, the ketone $1(2 \mathrm{mmol})$ dissolved in $1.5 \mathrm{~mL}$ of ethanol was added directly to the suspension where the yeasts grew. The resulting suspension was stirred in an orbital shaker $(200 \mathrm{rpm})$ at $28^{\circ} \mathrm{C}$ for G. candidum and at $30^{\circ} \mathrm{C}$ for $R$. glutinis until the full conversion of $\mathbf{1}(18 \mathrm{~h})$. The product was extracted with $\mathrm{CH}_{2} \mathrm{Cl}_{2}$ and purified by flash silica gel column chromatography using hexane/ethyl acetate $(7: 3)$.

2.3. (S)-(+)-2-Bromo-1-(4-bromophenyl)ethanol(S)-2a. The bioreduction of ketone $1 \mathrm{a}(0.556 \mathrm{~g}, 2 \mathrm{mmol})$ by Geotrichum candidum CCT 1205 furnished (S)-2a (0.540 g with 96.4\%) as colorless solid, m.p. $72^{\circ} \mathrm{C} ;[\alpha]_{D}^{25}+40.0^{\circ}\left(c 1, \mathrm{CHCl}_{3}\right)$ [lit. $-31.0^{\circ}, c 2.9, \mathrm{CHCl}_{3}$ for $\mathrm{R}$ isomer, $94 \%$ ee] [31], giving an optical purity of $>99 \%$ determined by GC using a chiral column; IR (KBr): 3402, 3086, 3064, 3049, 3026, 2958, 2922, $2852,1593,1488,1420,1402,1218,1192,1071,1010,828$, $722,680,613 \mathrm{~cm}^{-1} ;{ }^{1} \mathrm{H}$ NMR $\left(300 \mathrm{MHz}, \mathrm{CDCl}_{3}\right): \delta 2.74(\mathrm{~s}$, $1 \mathrm{H}, \mathrm{OH}), 3.46\left(\mathrm{dd}, 1 \mathrm{H}, J=8.4 \mathrm{~Hz}\right.$ and $\left.11.3 \mathrm{~Hz}, \mathrm{CH}_{2}\right), 3.59$ $\left(\mathrm{dd}, 1 \mathrm{H}, J=3.6 \mathrm{~Hz}\right.$ and $\left.11.3 \mathrm{~Hz}, \mathrm{CH}_{2}\right), 4.87(\mathrm{dd}, 1 \mathrm{H}, J=$ $3.6 \mathrm{~Hz}$ and $8.4 \mathrm{~Hz}, \mathrm{CH}), 7.24-7.31(\mathrm{~m}, 2 \mathrm{H}, \mathrm{Ph}), 7.48-7.51$ $(\mathrm{m}, 2 \mathrm{H}, \mathrm{Ph}) ;{ }^{13} \mathrm{C}$ NMR $\left(75 \mathrm{MHz}, \mathrm{CDCl}_{3}\right): \delta 39.72,72.97$, 122.12, 127.43, 131.55, 138.99; MS m/z (rel. int. \%): 188 (5), 187 (71), 186 (7), 185 (79), 183 (4), 182 (2), 171 (2), 169 (2), 159 (13), 158 (2), 157 (17), 155 (4), 120 (4), 119 (2), $106(3)$, 105 (6), 103 (4), 102 (9), 91 (14), 90 (6), 89 (7), 79 (6), 77 (100), 75 (18), 78 (46), 76 (16), 50 (62), 51 (57), 43 (39).

2.4. (R)-(-)-2-Bromo-1-(4-bromophenyl)ethanol (R)-2a. The bioreduction of ketone $1 \mathrm{a}(0.556 \mathrm{~g}, 2 \mathrm{mmol})$ by Rhodotorula glutinis CCT 2182 furnished $(R)-2 \mathrm{a}(0.554 \mathrm{~g}, 99.0 \%$ yield) as colorless solid, m.p. $72^{\circ} \mathrm{C} ;[\alpha]_{D}^{25}-40.4^{\circ}\left(c 1, \mathrm{CHCl}_{3}\right)[$ lit. $-31.0^{\circ}, c 2.9, \mathrm{CHCl}_{3}$ for $\mathrm{R}$ isomer, $94 \%$ ee] [31], giving an optical purity of $>99 \%$ determined by GC using a chiral column; ${ }^{1} \mathrm{H}$ and ${ }^{13} \mathrm{C}$ NMR and IR spectra and MS analysis were identical to those observed with its $(S)$ enantiomer.

2.5. (S)-(+)-2-Bromo-1-(4-chlorophenyl)ethanol (S)-2b. The bioreduction of ketone $\mathbf{1 b}(0.467 \mathrm{~g}, 2 \mathrm{mmol})$ by Geotrichum candidum CCT 1205 furnished (S)-2b (0.448 g, 95.1\% yield) as colorless oil; $[\alpha]_{D}^{25}+38.7^{\circ}\left(c 1, \mathrm{CHCl}_{3}\right)$ [lit. $38.6^{\circ}, c 1.15$, $\mathrm{CHCl}_{3}$ for $\mathrm{S}$ isomer, $91 \%$ ee] [32], giving an optical purity of $>99 \%$ determined by GC using a chiral column; IR (film): 3392 , 3088, 3051, 3030, 3003, 2957, 2896, 1596, 1492, 1428, $1408,1338,1310,1256,1310,1256,1199,1173,1089,1072$, $1013,973,944,897,834,778,752,704,674 \mathrm{~cm}^{-1}$; ${ }^{1} \mathrm{H}$ NMR 
$\left(300 \mathrm{MHz}, \mathrm{CDCl}_{3}\right) \delta 2.74(\mathrm{sl}, 1 \mathrm{H}, \mathrm{OH}), 3.48(\mathrm{dd}, 1 \mathrm{H}, J=$ $7.0 \mathrm{~Hz}$ and $\left.11.3 \mathrm{~Hz}, \mathrm{CH}_{2}\right), 3.61(\mathrm{dd}, 1 \mathrm{H}, J=5.8 \mathrm{~Hz}$ and $\left.11.3 \mathrm{~Hz}, \mathrm{CH}_{2}\right), 4.87(\mathrm{dd}, 1 \mathrm{H}, J=5.8 \mathrm{~Hz}$ and $7.0 \mathrm{~Hz}, \mathrm{CH})$, 7.24-7.28 (m, 2H, Ph), 7.31-7.51 (m, 2H, Ph); ${ }^{13} \mathrm{C}$ NMR $\left(75 \mathrm{MHz}, \mathrm{CDCl}_{3}\right) \delta 39.72,72.97,122.12,127.43,131.56$, 139.00; MS m/z (rel. int. \%): 143 (29), 142 (11), 141 (100), 139 (4), 138 (2), 121 (7), 115 (6), 113 (16), 112 (6), 111 (4), 108 (7), 107 (5), 105 (4), 104 (2), 103 (2), 102 (2), 91 (2), 89 (1), 79 (11), 78 (9), 77 (70), 76 (2), 75 (15), 74 (9), 70 (6), 63 (8), 51 (28), 50 (30), 49 (3), 44 (22), 43 (20), 40 (32).

2.6. (R)-(-)-2-Bromo-1-(4-chlorophenyl)ethanol (R)-2b. The bioreduction of ketone $\mathbf{1 b}(0.467 \mathrm{~g}, 2 \mathrm{mmol})$ by Rhodotorula glutinis CCT 2182 furnished $(R)-2 b(0.457 \mathrm{~g}, 97.0 \%$ yield $)$ as colorless oil; $[\alpha]_{D}^{25}-38.7\left(c 1, \mathrm{CHCl}_{3}\right)$ [lit. 38.6, c 1.15, $\mathrm{CHCl}_{3}$ for $\mathrm{S}$ isomer, $91 \%$ ee] [32], giving an optical purity of $>99 \%$ determined by GC using a chiral column; ${ }^{1} \mathrm{H}$ and ${ }^{13} \mathrm{C}$ NMR and IR spectra and MS analysis were identical to those observed with its $(S)$ enantiomer.

2.7. (S)-(+)-2-Bromo-1-(4-methylphenyl)ethanol (S)-2c. The bioreduction of ketone $1 \mathrm{c}(0.426 \mathrm{~g}, 2 \mathrm{mmol})$ by Geotrichum candidum CCT 1205 furnished $(S)-2 c(0.413 \mathrm{~g}, 96.0 \%$ yield $)$ as colorless oil; $[\alpha]_{D}^{25}+48.3^{\circ}\left(c 1, \mathrm{CHCl}_{3}\right)$ [lit. $+41.8^{\circ}, c 1.0$, $\mathrm{CHCl}_{3}$ for $\mathrm{S}$ isomer, 95\% ee] [32,33], giving an optical purity of $>99 \%$ determined by GC using a chiral column; IR (film): 3378, 3064, 3044, 2971, 2931, 2907, 2836, 1612, 1585, $1511,1458,1443,1368,1300,1243,1205,1174,1115,1087$, $1069,1034,1004,898,830,807 \mathrm{~cm}^{-1}$; ${ }^{1} \mathrm{H}$ NMR $(300 \mathrm{MHz}$, $\left.\mathrm{CDCl}_{3}\right) \delta 2.32\left(\mathrm{~s}, 3 \mathrm{H}, \mathrm{CH}_{3}\right), 2.46(\mathrm{sl}, 1 \mathrm{H}, \mathrm{OH}), 3.50(\mathrm{dd}$, $1 \mathrm{H}, J=8.7 \mathrm{~Hz}$ and $\left.10.4 \mathrm{~Hz}, \mathrm{CH}_{2}\right), 3.62(\mathrm{dd}, 1 \mathrm{H}, J=3.4 \mathrm{~Hz}$ and $\left.10.4 \mathrm{~Hz}, \mathrm{CH}_{2}\right), 4.87(\mathrm{dd}, 1 \mathrm{H}, J=3.4 \mathrm{~Hz}$ and $8.7 \mathrm{~Hz}, \mathrm{CH})$, $7.18-7.26(\mathrm{~d}, 2 \mathrm{H}, J=8 \mathrm{~Hz}, \mathrm{Ph}), 7.30(\mathrm{~d}, 2 \mathrm{H}, J=8.0 \mathrm{~Hz}, \mathrm{Ph})$; ${ }^{13} \mathrm{C}$ NMR $\left(75 \mathrm{MHz}, \mathrm{CDCl}_{3}\right) \delta 21.16,40.12,73.67,125.91$, 129.32, 137.32, 138.16; MS m/z (rel. int. \%): 217-215 ( $\mathrm{M}^{+}$, 2-2), 202 (2), 200 (2), 138 (7), 137 (93), 136 (16), 135 (13), 134 (45), 123 (1), 122 (2), 121 (2), 120 (3), 119 (5), 118 (5), 117 (8), 115 (4), 110 (4), 109 (49), 108 (4), 107 (4), 105 (4), 104 (2), 103 (4), 102 (2), 95 (3), 94 (34), 93 (43), 92 (9), 91 (38), 90 (2), 89 (5), 81 (2), 79 (9), 78 (8), 77 (30), 76 (4), 75 (3), 74 (4), 68 (5), 67 (3), 66 (13), 65 (20), 64 (11), 63 (21), 62 (8), 61 (3), 55 (4), 54 (1), 53 (10), 52 (8), 51 (30), 50 (19), 49 (1), 45 (8), 44 (6), 43 (100), 41 (12), 40 (10).

2.8. (R)-(-)-2-Bromo-1-(4-methylphenyl)ethanol (R)-2c. The bioreduction of ketone $1 \mathrm{c}(0.426 \mathrm{~g}, 2 \mathrm{mmol})$ by Rhodotorula glutinis CCT 2182 furnished $(R)-2 \mathrm{c}(0.410 \mathrm{~g}, 95.3 \%$ yield $)$ as colorless oil; $[\alpha]_{D}^{25}-48.3\left(c 1, \mathrm{CHCl}_{3}\right)$ [lit. $+41.8^{\circ}, c 1$, $\mathrm{CHCl}_{3}$ for $S$ isomer] [32], giving an optical purity of $>99 \%$ determined by GC using a chiral column; ${ }^{1} \mathrm{H}$ and ${ }^{13} \mathrm{C}$ NMR and IR spectra and MS analysis were identical to those observed with its $(S)$ enantiomer.

2.9. (S)-(+)-2-Bromo-1-(4-methoxyphenyl)ethanol (S)-2d. The bioreduction of ketone 1d $(0.458 \mathrm{~g}, 2 \mathrm{mmol})$ by Geotrichum candidum CCT 1205 gave $(S)-2 d(0.453 \mathrm{~g}, 98.0 \%$ yield) as colorless oil; $[\alpha]_{D}^{25}+19.8^{\circ}\left(c 1, \mathrm{CHCl}_{3}\right)$ [lit. $-37.7^{\circ}$, c $1.0, \mathrm{CHCl}_{3}$ for $\mathrm{R}$ isomer, $87 \%$ ee] [31, 34], IR (film): 3371 ,
3062, 3030, 2973, 2928, 2907, 2878, 1616, 1581, 1511, 1458, $1442,1368,1300,1240,1205,1174,1112,1081,1069,1024$, $1001,892,830,804 \mathrm{~cm}^{-1} ;{ }^{1} \mathrm{H} \mathrm{NMR}\left(300 \mathrm{MHz}, \mathrm{CDCl}_{3}\right) \delta 2.72$ $(\mathrm{sl}, 1 \mathrm{H}, \mathrm{OH}), 3.61\left(\mathrm{dd}, 1 \mathrm{H}, J=8.7 \mathrm{~Hz}\right.$ and $\left.11.2 \mathrm{~Hz}, \mathrm{CH}_{2}\right)$, $3.70\left(\mathrm{dd}, J=3.9 \mathrm{~Hz}\right.$ and $\left.11.2 \mathrm{~Hz}, \mathrm{CH}_{2}\right), 3.79\left(\mathrm{~s}, 3 \mathrm{H}, \mathrm{CH}_{3}\right)$, $4.86(\mathrm{dd}, 1 \mathrm{H}, J=3.9 \mathrm{~Hz}$ and $8.7 \mathrm{~Hz}, \mathrm{CH}), 6.89(\mathrm{~d}, 2 \mathrm{H}, J=$ $8.8 \mathrm{~Hz}, \mathrm{Ph}), 7.31(\mathrm{~d}, 2 \mathrm{H}, J=8.8 \mathrm{~Hz}, \mathrm{Ph}) ;{ }^{13} \mathrm{C} \mathrm{NMR}(75 \mathrm{MHz}$, $\left.\mathrm{CDCl}_{3}\right) \delta 42.12,56.28,78.95,114.94,127.71,133.40,160.03$; MS $m / z$ (rel. int. \%): 233-231 $\left(\mathrm{M}^{+}, 1-1\right), 218$ (1), 215 (1), 214 (1), 202 (2), 200 (2), 153 (2), 152 (4), 151 (2), 138 (6), 137 (100), 135 (11), 134 (9), 122 (2), 121 (2), 120 (2), 119 (20), 110 (3), 109 (16), 108 (3), 107 (2), 105 (2), 104 (1); 103 (4), 102 (2), 95 (3), 94 (16), 93 (2), 92 (7), 91(18), 90 (2), 89 (4), 81 (2), 79 (6), 78 (5), 77 (21), 76 (2), 75 (3), 68 (2), 67 (2), 66 (5), 65 (12), 64 (9), 63 (6), 55 (1), 54 (1), 53 (8), 52 (4), 51 (12), 50 (14), 45 (3), 44 (2), 43 (79), 41 (10), 40 (8).

2.10. (R)-(-)-2-Bromo-1-(4-methoxyphenyl)ethanol (R)-2d. The bioreduction of ketone $1 \mathbf{d}(0.458 \mathrm{~g}, 2 \mathrm{mmol})$ by Rhodotorula glutinis CCT 2182 gave $(R)-2 d(0.452 \mathrm{~g}, 97.8 \%$ yield) as colorless oil; $[\alpha]_{D}^{25}-19.7\left(c 1, \mathrm{CHCl}_{3}\right)$ [lit. $-37.7^{\circ}, c 1.0$, $\mathrm{CHCl}_{3}$ for $\mathrm{R}$ isomer, $87 \%$ ee] [31], ${ }^{1} \mathrm{H}$ and ${ }^{13} \mathrm{C}$ NMR and IR spectra and MS analysis were identical to those observed with its $(S)$ enantiomer.

2.11. (S)-(+)-2-Bromo-1-(4-nitrophenyl)ethanol (S)-2e. The bioreduction of ketone $1 \mathrm{e}(0.488 \mathrm{~g}, 2 \mathrm{mmol})$ by Geotrichum candidum CCT 1205 gave $(S)-2 e(0.480 \mathrm{~g}, 97.6 \%$ yield $)$ a light yellow solid, mp $98^{\circ} \mathrm{C} ;[\alpha]_{D}^{25}+25.0^{\circ}\left(c 1, \mathrm{CHCl}_{3}\right)\left[\right.$ lit. $+32.1^{\circ}$, c $1, \mathrm{CHCl}_{3}$ for $\mathrm{S}$ isomer, $91 \%$ ee] [33,35], giving an optical purity of $>99 \%$ determined by GC using a chiral column. IR (KBr): 3455, 3109, 3079, 2947, 2924, 2889, 2851, 1601, $1520,1347,1291,1203,1074,1012,855,760,730 \mathrm{~cm}^{-1} ;{ }^{1} \mathrm{H}$ NMR $\left(300 \mathrm{MHz}, \mathrm{CDCl}_{3}\right): \delta 2.83(\mathrm{sl}, 1 \mathrm{H}, \mathrm{OH}), 3.53(\mathrm{dd}$, $1 \mathrm{H}, J=8.4 \mathrm{~Hz}$ and $\left.10.6 \mathrm{~Hz}, \mathrm{CH}_{2}\right), 3.68(\mathrm{dd}, 1 \mathrm{H}, J=3.5 \mathrm{~Hz}$ and $\left.10.6 \mathrm{~Hz}, \mathrm{CH}_{2}\right), 5.03-5.08(\mathrm{~m}, 1 \mathrm{H}, \mathrm{CH}), 7.45(\mathrm{~d}, 2 \mathrm{H}, J=$ $8.8 \mathrm{~Hz}, \mathrm{Ph}), 8.22(\mathrm{~d}, 2 \mathrm{H}, J=8.8 \mathrm{~Hz}, \mathrm{Ph}),{ }^{13} \mathrm{C}$ NMR $(75 \mathrm{MHz}$, $\left.\mathrm{CDCl}_{3}\right): \delta 39.32,72.52,123.60,126.65,146.10,146.90$; MS $\mathrm{m} / \mathrm{z}$ (rel. int. \%): 153 (8), 152 (100), 149 (2), 141 (1), 139 (1), 136 (2), 127 (1), 125 (2), 122 (5), 106 (10), 105 (9), 102 (4), 95 (5), 94 (11), 91 (8), 78 (13), 77 (17), 66 (6), 51 (17), 50 (13), $43(20)$.

2.12. (R)-(-)-2-Bromo-1-(4-nitrophenyl)ethanol (R)-2e. The bioreduction of ketone $1 \mathrm{e}(0.488 \mathrm{~g}, 2 \mathrm{mmol})$ by Rhodotorula glutinis CCT 2182 gave $(R)-2 \mathrm{e}(0.483 \mathrm{~g}, 98.0 \%$ yield $)$ a light yellow solid, mp $98^{\circ} \mathrm{C} ;[\alpha]_{D}^{25}-25.0^{\circ}\left(c 1, \mathrm{CHCl}_{3}\right)\left[\right.$ lit. $+32.1^{\circ}$, $c 1, \mathrm{CHCl}_{3}$ for $S$ isomer, $91 \%$ ee] $[33,36]$, giving an optical purity of $>99 \%$ determined by GC using a chiral column; ${ }^{1} \mathrm{H}$ and ${ }^{13} \mathrm{C}$ NMR and IR spectra and MS analysis were identical to those observed with its $(S)$ enantiomer.

2.13. (S)-(+)-2-Chloro-1-(4-bromophenyl)ethanol(S)-2f. The bioreduction of ketone 1f $(0.467 \mathrm{~g}, 2 \mathrm{mmol})$ by Geotrichum candidum CCT 1205 gave $(S)-2 \mathrm{f}(0.468 \mathrm{~g}, 99.4 \%$ yield $)$ as colorless oil; $[\alpha]_{D}^{25}+35.0^{\circ}\left(c 1, \mathrm{CHCl}_{3}\right)\left[\right.$ lit. $-35.87^{\circ}, c 1.1072$, $\mathrm{CHCl}_{3}$ for $\mathrm{R}$ isomer, $99 \%$ ee] [14], giving an optical purity of $>99 \%$ determined by GC using a chiral column; IR (film): 
$3421 ; 3106 ; 3087 ; 3064 ; 3031 ; 3006 ; 2956 ; 2895 ; 1593,1575$, $1494 ; 1453 ; 1426 ; 1387 ; 1336 ; 1300 ; 1295 ; 1248 ; 1200 ; 1085$; $1064 ; 1074 ; 1030 ; 1012 ; 972 ; 944 ; 917 ; 869 ; 824 ; 768 ; 724$; $698 \mathrm{~cm}^{-1} ;{ }^{1} \mathrm{H}$ NMR $\left(300 \mathrm{MHz}, \mathrm{CDCl}_{3}\right): \delta 2.41(\mathrm{sl}, 1 \mathrm{H}$, $\mathrm{OH}), 3.61\left(\mathrm{dd}, 1 \mathrm{H}, J=8.7 \mathrm{~Hz}\right.$ and $\left.11.3 \mathrm{~Hz}, \mathrm{CH}_{2}\right), 3.73(\mathrm{dd}$, $1 \mathrm{H}, J=3.4 \mathrm{~Hz}$ and $\left.11.3 \mathrm{~Hz}, \mathrm{CH}_{2}\right), 4.88(\mathrm{dd}, 1 \mathrm{H}, J=3.4 \mathrm{~Hz}$ and $8.7 \mathrm{~Hz}, \mathrm{CH}), 7.28(\mathrm{~d}, 2 \mathrm{H}, J=8.4 \mathrm{~Hz}, \mathrm{Ph}), 7.51(\mathrm{~d}, 2 \mathrm{H}$, $J=8.4 \mathrm{~Hz}, \mathrm{Ph}) ;{ }^{13} \mathrm{C} \mathrm{NMR}\left(75 \mathrm{MHz}, \mathrm{CDCl}_{3}\right): \delta 50.90,74.12$, 122.04, 127.28, 132.02, 139.46; MS m/z (rel. int. \%): 238-236 $\left(\mathrm{M}^{+}, 1-1\right), 202$ (1), 200 (1), 158 (1), 156 (3), 108 (7), 107 (100), 105 (5), 104 (2), 103 (4), 102 (1), 91 (6), 89 (1), 79 (60), 78 (8), 77 (42), 76 (2), 75 (2), 51 (31), 50 (13).

2.14. (R)-(-)-2-Chloro-1-(4-bromophenyl)ethanol (R)-2f. The bioreduction of ketone 1 f $(0.467 \mathrm{~g}, 2 \mathrm{mmol})$ by Rhodotorula glutinis CCT 2182 gave $(R)$-2f $(0.460$ g, 97.7\% yield) as colorless oil; $[\alpha]_{D}^{25}-34.9\left(c 1, \mathrm{CHCl}_{3}\right)\left[\right.$ lit. $-35.87^{\circ}$, c $1.1072, \mathrm{CHCl}_{3}$ for $R$ isomer, $99 \%$ ee] [14], giving an optical purity of $>99 \%$ determined by GC using a chiral column; ${ }^{1} \mathrm{H}$ and ${ }^{13} \mathrm{C}$ NMR and IR spectra and MS analysis were identical to those observed with its $(S)$ enantiomer.

2.15. (S)-(+)-2-Chloro-1-(4-chlorophenyl)ethanol (S)-2g. The bioreduction of ketone $1 \mathrm{~g}(0.378 \mathrm{~g}, 2 \mathrm{mmol})$ by Geotrichum candidum CCT 1205 furnished $(S)-2 \mathrm{~g}(0.363 \mathrm{~g}, 95.0 \%$ yield $)$ as colorless oil; $[\alpha]_{D}^{25}+48.3^{\circ}\left(c 1.25, \mathrm{CHCl}_{3}\right)\left[\right.$ lit. $[\alpha]_{D}^{20} 44.2^{\circ}$ (c 2.1, $\mathrm{CHCl}_{3}$ ) for $S$ isomer, 96,6\% ee] [36], giving an optical purity of $>99 \%$ determined by GC using a chiral column; IR (film): 3387, 3103, 3090, 3067, 3053, 3020, 2956, 2894, $1598,1492,1427,1410,1338,1308,1252,1198,1090,1075$, $1013,970,947,895,871,833,776,751,704,673 \mathrm{~cm}^{-1} ;{ }^{1} \mathrm{H}$ $\mathrm{NMR}\left(300 \mathrm{MHz}, \mathrm{CDCl}_{3}\right): \delta 3.2(\mathrm{sl}, 1 \mathrm{H}, \mathrm{OH}), 3.58(\mathrm{dd}, 1 \mathrm{H}, J$ $=8.4 \mathrm{~Hz}$ and $\left.11.3 \mathrm{~Hz}, \mathrm{CH}_{2}\right), 3.67(\mathrm{dd}, 1 \mathrm{H}, J=3.7 \mathrm{~Hz}$ and $\left.11.3 \mathrm{~Hz}, \mathrm{CH}_{2}\right), 4.84(\mathrm{dd}, 1 \mathrm{H}, J=3.7 \mathrm{~Hz}$ and $8.4 \mathrm{~Hz}, \mathrm{CH})$, 7.27-7.34 (m, 4H, Ph); $\left.{ }^{13} \mathrm{C} \mathrm{NMR} \mathrm{(75} \mathrm{MHz,} \mathrm{CDCl}_{3}\right): \delta 50.39$, 73.15, 127.16, 128.27, 128.48, 133.82, 138.09; MS $\mathrm{m} / z$ (rel. int. \%): 192-191 (M+, 4), 158 (2), 156 (7), 143 (11), 142 (3), 141 (38), 139 (4), 138 (2), 121 (7), 115 (6), 114 (3), 113 (19), 112 (6), 111 (5), 105 (2), 103 (5), 102 (2), 101 (1), 91 (2), 89 (1), 87 (1), 85 (2), 78 (8), 77 (77), 75 (13), 74 (7), 73 (3), 70 (7), 71 (2), 65 (2), 63 (5), 62 (3), 61 (2), 60 (1), 55 (1), 53 (3), 52 (6), 51 (33), 50 (23), 49 (3), 46 (1), 45 (11), 44 (4), 42 (100), 41 (1), 40 (1).

2.16. (R)-(-)-2-Cloro-1-(4-chlorophenyl)ethanol ( $R)-2 g$. The bioreduction of ketone $1 \mathrm{~g}(0.378 \mathrm{~g}, 2 \mathrm{mmol})$ by Rhodotorula glutinis CCT 2182 furnished $(R)-2 \mathrm{~g}(0.36 \mathrm{~g}, 94.2 \%$ yield $)$ as colorless oil; $[\alpha]_{D}^{25}-48.3^{\circ}$ (c 2.1, $\mathrm{CHCl}_{3}$ ); [lit. $[\alpha]_{D}^{20} 44.2^{\circ}$ (c $2.1, \mathrm{CHCl}_{3}$ ) for $S$ isomer, $96,6 \%$ ee] [36], giving an optical purity of $>99 \%$ determined by GC using a chiral column; ${ }^{1} \mathrm{H}$ and ${ }^{13} \mathrm{C}$ NMR and IR spectra and MS analysis were identical to those observed with its $(S)$ enantiomer.

2.17.(S)-(+)-2-Chloro-1-(4-methylphenyl)ethanol(S)-2h. The bioreduction of ketone $\mathbf{1 h}(0.337 \mathrm{~g}, 2 \mathrm{mmol})$ by Geotrichum candidum CCT 1205 furnished $(S)-2 h(0.329$ g, 96.4\% yield) as colorless oil; $[\alpha]_{D}^{25}+48.3^{\circ}\left(c\right.$ 1.1, $\left.\mathrm{CHCl}_{3}\right)\left[\right.$ lit. $+47.2^{\circ}(c$ $\left.1.1, \mathrm{CHCl}_{3}\right)$ for $S$ isomer, 92\% ee] [32], giving an optical purity of $>99 \%$ determined by GC using a chiral column; IR (film): 3414, 3094, 3052, 3017, 2970, 2924, 2863, 1611, 1512, $1445,1411,1369,1302,1280,1257,1192,1181,1112,1090$, 1071, 1010, 941, 892, 813, $724 \mathrm{~cm}^{-1} ;{ }^{1} \mathrm{H} \mathrm{NMR}(300 \mathrm{MHz}$, $\left.\mathrm{CDCl}_{3}\right): \delta 2,34\left(\mathrm{~s}, 3 \mathrm{H}, \mathrm{CH}_{3}\right), 2.50(\mathrm{sl}, 1 \mathrm{H}, \mathrm{OH}), 3.63(\mathrm{dd}$, $1 \mathrm{H}, J=8.5 \mathrm{~Hz}$ and $\left.11.2 \mathrm{~Hz}, \mathrm{CH}_{2}\right), 3.74(\mathrm{dd}, 1 \mathrm{H}, J=3.9 \mathrm{~Hz}$ and $\left.11.2 \mathrm{~Hz}, \mathrm{CH}_{2}\right), 4.85(\mathrm{dd}, 1 \mathrm{H}, J=3.9$ and $8.5 \mathrm{~Hz}, \mathrm{CH})$, $7.20(\mathrm{~d}, 2 \mathrm{H}, J=8 \mathrm{~Hz}, \mathrm{Ph}), 7.29(\mathrm{~d}, 2 \mathrm{H}, J=8 \mathrm{~Hz}, \mathrm{Ph})$; ${ }^{13} \mathrm{C} \mathrm{NMR}\left(75 \mathrm{MHz}, \mathrm{CDCl}_{3}\right): \delta 21.17,50.82,73.98,126.05$, 129.39, 137.13, 138.22; MS m/z (rel. int. \%): 171-170 $\left(\mathrm{M}^{+}, 4\right)$ 158 (1), 156 (3), 137 (2), 136 (16), 135 (1), 122 (4), 121 (50), 119 (5), 118 (5),117 (8), 115 (4), 107 (2), 105 (1), 103 (1), 102 (1), 94 (4), 93 (49), 92 (11), 91 (45), 89 (4), 78 (5), 79 (2), 77 (30), 75 (1), 74 (1), 67 (3), 66 (2), 65 (20), 64 (2), 63 (10), 62 (4), 60 (15), 59 (2), 57 (4), 55 (1), 53 (4), 52 (5), 51 (18), 50 (9), 46 (1), 45 (9), 44 (3), 43 (100), 41 (10), 40 (4).

2.18. (R)-(-)-2-Chloro-1-(4-methylphenyl)ethanol (R)-2h. The bioreduction of ketone $1 \mathbf{h}(0.337 \mathrm{~g}, 2 \mathrm{mmol})$ by Rhodotorula glutinis CCT 2182 furnished $(R)-2 \mathrm{~h}(0.327 \mathrm{~g}$, 95.7\% yield) as colorless oil; $[\alpha]_{D}^{25}-48.3$ ( c 1.1, $\left.\mathrm{CHCl}_{3}\right)$ [lit. $+47.2^{\circ}$ ( $c$ 1.1, $\mathrm{CHCl}_{3}$ ) for $S$ isomer, 92\% ee] [32], giving an optical purity of $>99 \%$ determined by GC using a chiral column; ${ }^{1} \mathrm{H}$ and ${ }^{13} \mathrm{C}$ NMR and IR spectra and MS analysis were identical to those observed with its $(S)$ enantiomer.

2.19. (S)-(+)-2-Chloro-1-(4-methoxyphenyl)ethanol (S)-2i. The bioreduction of ketone $1 \mathbf{i}(0.369 \mathrm{~g}, 2 \mathrm{mmol})$ by Geotrichum candidum CCT 1205 furnished (S)-2i $(0.370 \mathrm{~g}$, 99.2\% yield) as colorless oil; $[\alpha]_{D}^{25}+41.4^{\circ}$ (c 1, $\left.\mathrm{CHCl}_{3}\right)$ [lit. $+40.2^{\circ}$, for $S$ isomer, $90,5 \%$ ee] [36], giving an optical purity of $>99 \%$ determined by GC using a chiral column; IR (film): 3400, 3372, 3062, 3031, 2950, 2931, 2907, 2836, 1610, 1520, $1511,1458,1443,1368,1300,1250,1205,1174,1115,1084$, $1069,1030,1004,898 ; 840,780 \mathrm{~cm}^{-1} ;{ }^{1} \mathrm{H}$ NMR $(300 \mathrm{MHz}$, $\left.\mathrm{CDCl}_{3}\right) \delta 2.70(\mathrm{sl}, 1 \mathrm{H}, \mathrm{OH}), 3.52(\mathrm{dd}, 1 \mathrm{H}, J=8.7 \mathrm{~Hz}$ and $\left.11.4 \mathrm{~Hz}, \mathrm{CH}_{2}\right), 3.61\left(\mathrm{dd}, 1 \mathrm{H}, J=3.8 \mathrm{~Hz}\right.$ and $\left.11.4 \mathrm{~Hz}, \mathrm{CH}_{2}\right)$, $3.80\left(\mathrm{~s}, 3 \mathrm{H}, \mathrm{CH}_{3}\right), 4.78(\mathrm{dd}, 1 \mathrm{H}, J=3.8 \mathrm{~Hz}$ and $8.7 \mathrm{~Hz}, \mathrm{CH})$, $6.90(\mathrm{~d}, J=8.7 \mathrm{~Hz}, 2 \mathrm{H}, \mathrm{Ph}), 7.20(\mathrm{~d}, J=8.7 \mathrm{~Hz}, 2 \mathrm{H}, \mathrm{Ph}) ;{ }^{13} \mathrm{C}$ $\operatorname{NMR}\left(75 \mathrm{MHz}, \mathrm{CDCl}_{3}\right) \delta 50.70,55.34,73.61,113.78,127.10$, 132.12, 159.62; MS $m / z$ (rel. int. \%): $186\left(\mathrm{M}^{+}, 6\right), 152(21)$, 153 (2), 151 (2), 138 (7), 137 (100), 135 (13), 134 (45), 122 (2), 121 (2), 120 (3), 119 (27), 110 (4), 109 (23), 108 (4), 107 (4), 105 (4), 104 (2), 103 (4), 102 (2), 95 (3), 94 (26), 93 (3), 92 (9), 90 (2), 91(38), 89 (5), 81 (2), 78 (8), 79 (9), 77 (25), 76 (4), 75 (3), 74 (4), 68 (5), 67 (3), 66 (13), 65 (38), 64 (11), 63 (21), 62 (8), 61 (3), 55 (4), 54 (1), 53 (10), 52 (8), 51 (30), 50 (19), 49 (1), 45 (8), 44 (6), 43 (100), 41 (12), 40 (10).

2.20. (R)-(-)-2-Chloro-1-(4-methoxyphenyl)ethanol (R)-2i. The bioreduction of ketone $1 \mathrm{i}(0.369 \mathrm{~g}, 2 \mathrm{mmol})$ by Rhodotorula glutinis CCT 2182 furnished $(R)-2 \mathbf{i}(0.366 \mathrm{~g}$, 98.0\% yield) as colorless oil; $[\alpha]_{D}^{25}-41.5$ (c 1, $\left.\mathrm{CHCl}_{3}\right)$ [lit. $+40.2^{\circ}$, for $S$ isomer, $90,5 \%$ ee] [36], giving an optical purity of $>99 \%$ determined by GC using a chiral column; ${ }^{1} \mathrm{H}$ and ${ }^{13} \mathrm{C}$ NMR and IR spectra and MS analysis were identical to those observed with its $(S)$ enantiomer. 
TABle 1: Asymmetric reduction of 2-halo-1-(4-substituted phenyl)-ethanones 1a-j mediated by Geotrichum candidum CCT 1205 and Rhodotorula glutinis CCT $2182^{\mathrm{a}}$.

\begin{tabular}{|c|c|c|c|c|c|}
\hline Ketone & Microorganism & $\mathrm{T}\left({ }^{\circ} \mathrm{C}\right)$ & Alcohol & Yield (\%) & {$[\alpha]_{\mathrm{D}}^{25^{b}}$} \\
\hline $1 \mathrm{a}$ & Geotrichum candidum & 28 & $(S)-2 \mathrm{a}$ & 96.4 & +40.0 \\
\hline $1 b$ & “ & 28 & $(S)-2 \mathrm{~b}$ & 95.1 & +38.7 \\
\hline $1 c$ & “ & 28 & $(S)-2 c$ & 96.0 & +48.3 \\
\hline 1d & “ & 28 & $(S)-2 \mathrm{~d}$ & 98.0 & +19.8 \\
\hline $1 \mathrm{e}$ & “ & 28 & $(S)-2 \mathrm{e}$ & 97.6 & +25.0 \\
\hline 1f & “ & 28 & $(S)-2 \mathrm{f}$ & 99.4 & +35.0 \\
\hline $1 g$ & “ & 28 & $(S)-2 \mathrm{~g}$ & 95.0 & +48.3 \\
\hline $1 \mathrm{~h}$ & “ & 28 & $(S)-2 \mathrm{~h}$ & 96.4 & +48.3 \\
\hline $1 \mathbf{i}$ & “ & 28 & $(S)-2 \mathbf{i}$ & 99.2 & +41.4 \\
\hline $1 \mathrm{j}$ & " & 28 & $(S)-2 \mathrm{j}$ & 97.0 & +32.6 \\
\hline $1 \mathrm{a}$ & Rhodotorula glutinis & 30 & $(R)-2 \mathrm{a}$ & 99.0 & -40.4 \\
\hline $1 b$ & “ & 30 & $(R)-2 b$ & 97.0 & -38.7 \\
\hline 1c & “ & 30 & $(S)-2 \mathrm{c}$ & 95.3 & -48.3 \\
\hline 1d & “ & 30 & $(R)-2 d$ & 97.8 & -19.7 \\
\hline $1 e$ & “ & 30 & $(R)-2 \mathrm{e}$ & 98.0 & -25.0 \\
\hline 1f & “ & 30 & $(R)-2 f$ & 97.7 & -34.9 \\
\hline $1 g$ & “ & 30 & $(R)-2 \mathrm{~g}$ & 94.2 & -48.3 \\
\hline $1 \mathrm{~h}$ & “ & 30 & $(R)-2 h$ & 95.7 & -48.3 \\
\hline $1 \mathrm{i}$ & “ & 30 & $(R)-2 \mathrm{i}$ & 98.0 & -41.5 \\
\hline $1 \mathrm{j}$ & " & 30 & $(R)-2 \mathrm{j}$ & 98.0 & -32.6 \\
\hline
\end{tabular}

${ }^{\mathrm{a}} 18 \mathrm{~h}, 2 \mathrm{mmol}$ of ketone $/ 1.5 \mathrm{~mL}$ of EtOH was added to $15 \mathrm{~g}$ of yeast (wet weight) $/ 400 \mathrm{~mL}$ of nutrient broth 1 (malt extract, peptone) for Geotrichum candidum

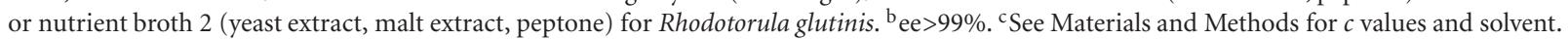

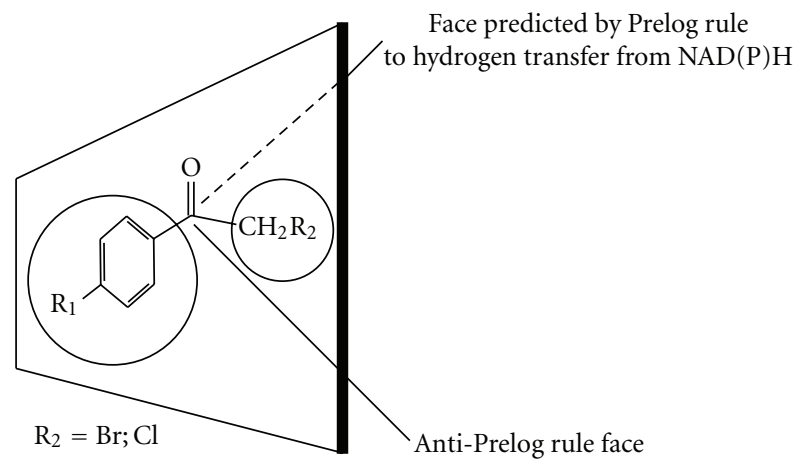

Figure 1: Prelog rule for discrimination of the faces of carbonylic group by the enzymes.

2.21. (S)-(+)-2-Chloro-1-(4-nitrophenyl)ethanol (S)-2j. The bioreduction of ketone $\mathbf{1 j}(0.399,2 \mathrm{mmol})$ by Geotrichum candidum CCT 1205 furnished $(S)-2 \mathbf{j}(0.391 \mathrm{~g}, 97.0 \%$ yield) a white solid, mp $87^{\circ} \mathrm{C}$ (lit. p.f. $87^{\circ} \mathrm{C}$ ) [33]; $[\alpha]_{D}^{25}+32,6^{\circ}(c$ $\left.1, \mathrm{CHCl}_{3}\right)$ [lit. $+37.2^{\circ}, c 2.0, \mathrm{CHCl}_{3}$ for $S$ isomer, $98,2 \%$ ee] [36], giving an optical purity of $>99 \%$ determined by GC using a chiral column; IR (KBr): 3304, 3051, 3021, 2970, 2923, 2878, 1599, 1506, 1452, 1364, 1323, 1275, 1251, 1204, $1166,1125,1075,1024,964,951,902,863,823,773,743$, 703, $652 \mathrm{~cm}^{-1} ;{ }^{1} \mathrm{H} \mathrm{NMR}\left(300 \mathrm{MHz}, \mathrm{CDCl}_{3}\right) \delta 2.93(\mathrm{sl}, 1 \mathrm{H}$, $\mathrm{OH}), 3.64\left(\mathrm{dd}, 1 \mathrm{H}, J=8.1 \mathrm{~Hz}\right.$ and $\left.11.3 \mathrm{~Hz}, \mathrm{CH}_{2}\right), 3.68(\mathrm{dd}$, $1 \mathrm{H}, J=3.3 \mathrm{~Hz}$ and $\left.11.3 \mathrm{~Hz}, \mathrm{CH}_{2}\right), 5.03-5.05(\mathrm{~m}, 1 \mathrm{H}, \mathrm{CH})$,
7.50 (d, 2H, $J=8.7 \mathrm{~Hz}, \mathrm{Ph}), 8.20(\mathrm{~d}, 2 \mathrm{H}, J=8.7 \mathrm{~Hz}, \mathrm{Ph}$ ); ${ }^{13} \mathrm{C}$ NMR $\left(75 \mathrm{MHz}, \mathrm{CDCl}_{3}\right) \delta 50.20,69.38,123.56,126.73$, 146.59, 146.95; MS m/z (rel. int. \%): 166 (5), 153 (8), 152 (100), 136 (2), 122 (6), 107 (2), 106 (13), 105 (12), 102 (3), 95 (4), 94 (13), 81 (3), 79 (3), 78 (18), 77 (22), 65 (9), 51 (24), 50 (16), 43 (23), 41 (9).

2.22. (R)-(-)-2-Chloro-1-(4-nitrophenyl)ethanol (R)-2j. The bioreduction of ketone $\mathbf{1 j}(0.399 \mathrm{~g}, 2 \mathrm{mmol})$ by Rhodotorula glutinis CCT 2182 furnished $(R)-2 \mathbf{j}(0.395 \mathrm{~g}$, $98.0 \%$ yield) a white solid, $\mathrm{mp} 87^{\circ} \mathrm{C}$ (lit. p.f. $87^{\circ} \mathrm{C}$ ) [36]; $[\alpha]_{D}^{25}-32,6^{\circ}\left(c 1, \mathrm{CHCl}_{3}\right)\left[\right.$ lit. $+37.2^{\circ}$, c 2.0, $\mathrm{CHCl}_{3}$ for $S$ isomer, $98,2 \%$ ee] [33], giving an optical purity of $>99 \%$ determined by GC using a chiral column; ${ }^{1} \mathrm{H}$ and ${ }^{13} \mathrm{C}$ NMR and IR spectra and MS analysis were identical to those observed with its $(S)$ enantiomer.

\section{Results and Discussion}

The reduction of ethanones $\mathbf{1} \mathbf{a}-\mathbf{j}$ was carried out in $5 \mathrm{mmol} / \mathrm{L}$ in a slurry of growing yeast of Rhodotorula glutinis CCT 2182 and Geotrichum candidum CCT 1205. These ethanones having substituted groups (electron withdrawing groupsEWG: $-\mathrm{NO}_{2},-\mathrm{Br},-\mathrm{Cl}$; electron donating groups-EDG: $\left.\mathrm{CH}_{3},-\mathrm{OCH}_{3}\right)$ attached to position 4 of benzene ring were studied in order to investigate the influence of these groups in the bioreduction performed by these two microorganisms. 
<smiles>[R]CC(=O)c1ccc([R])cc1C(=O)C[R2]</smiles><smiles>[R]c1ccc(C2CO2)cc1</smiles>

(S)-3

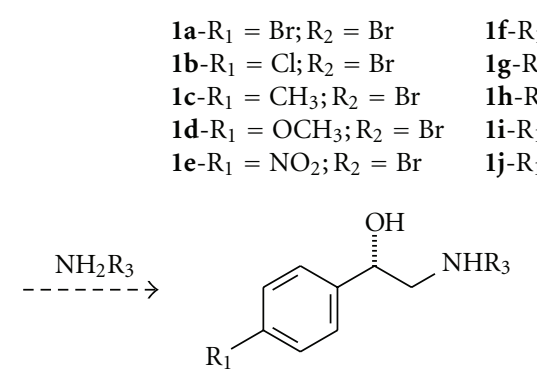

$(S)-4$

$$
\begin{aligned}
& \mathbf{1 f}-\mathrm{R}_{1}=\mathrm{Br} ; \mathrm{R}_{2}=\mathrm{Cl} \\
& \mathbf{1 g}-\mathrm{R}_{1}=\mathrm{Cl} ; \mathrm{R}_{2}=\mathrm{Cl} \\
& \mathbf{1 h}-\mathrm{R}_{1}=\mathrm{CH}_{3} ; \mathrm{R}_{2}=\mathrm{Cl} \\
& \mathbf{1 i}-\mathrm{R}_{1}=\mathrm{OCH}_{3} ; \mathrm{R}_{2}=\mathrm{Cl} \\
& \mathbf{1 j}-\mathrm{R}_{1}=\mathrm{NO}_{2} ; \mathrm{R}_{2}=\mathrm{Cl}
\end{aligned}
$$

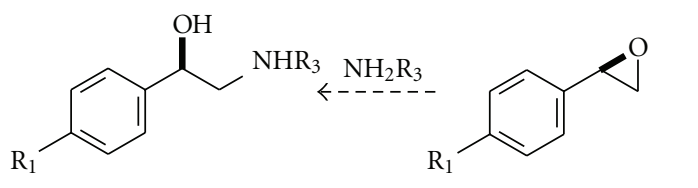

(R)-4
(R)-2 $\mathrm{NaOH} /$ Ether

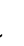

$(R)-3$

SCHeme 2

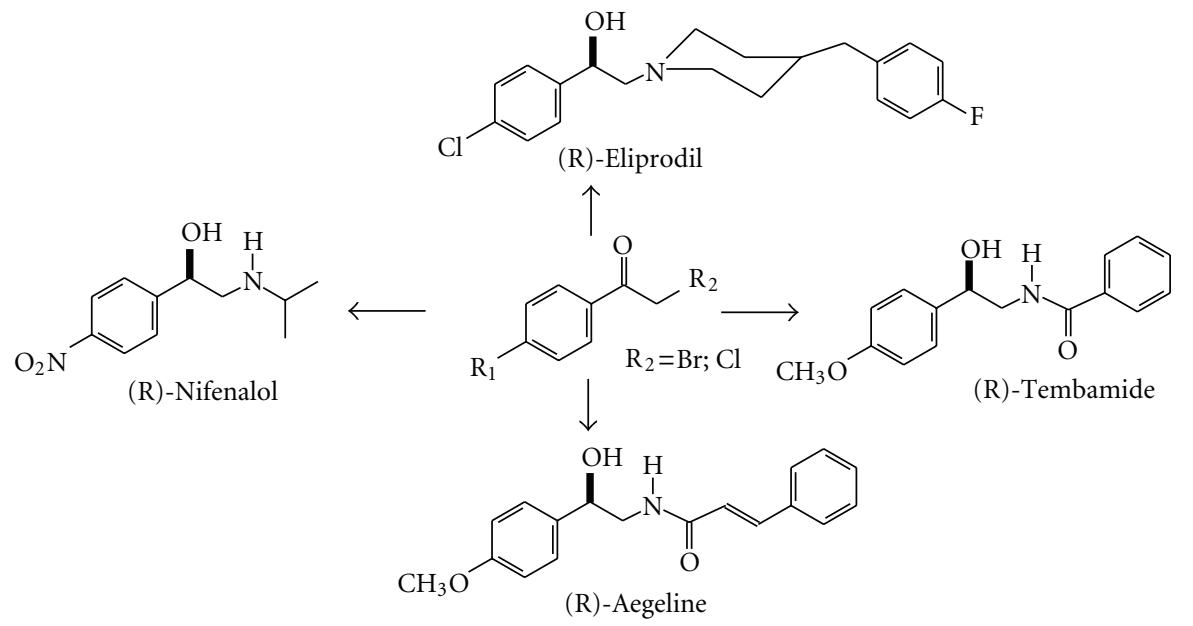

FIgURE 2: Pharmaceutical useful ethanolamines.

The reaction progress was monitored by GC analysis, and the yields and enatiomeric excesses are shown in Table 1.

The reductions of 2-bromo-1-(4-substituted phenyl)ethanones 1a-e and 2-chloro-1-(4-substituted)-ethanones 1f-j mediated by Rhodotorula glutinis CCT 2182 gave the corresponding halohydrins $\mathbf{2} \mathbf{a}-\mathbf{j}$ with $(R)$ configuration, while the halohydrins $\mathbf{2} \mathbf{a}-\mathbf{j}$ with $(S)$ configuration were obtained when Geotrichum candidum CCT 1205 mediated the reduction of the ethanones $\mathbf{1 a - j}$.

$\alpha$-Haloacetophenones have been used as mechanistic probe in the reduction reactions of NADH-dependent horse liver alcohol dehydrogenase [37-40], for identification of reductants in sediments [41] and even in the whole cells [42]. This probe enables the differentiation between reduction processes which proceed through hydride transfer $\left(\mathrm{H}^{-}\right)$ or by a multistep electron transfer $\left(\mathrm{e}^{-}, \mathrm{H}^{\bullet}\right.$ or $\mathrm{e}^{-}, \mathrm{H}^{+}$, $\mathrm{e}^{-}$as has been suggested). Acetophenone is the reduction product obtained by electron transfer, while optically active halohydrin is obtained when an enzyme mediates a hydride transfer process. In this work, the reductions of 1a-e proceed via hydride transfer mediated by an oxireductase, since halohydrins were obtained in high ee and no 4-substituted acetophenone was detected.

Rhodotorula glutinis gives products following the Prelog rule [43], which predicts that, in general, hydrogen transfer from $\mathrm{NAD}(\mathrm{P}) \mathrm{H}$ to the prochiral ethanones $\mathbf{1} \mathbf{a}-\mathbf{j}$ occurs to the face of carbonylic group shown in Figure 1, taking into account that the aryl group is larger than the $-\mathrm{CH}_{2} \mathrm{Br}$ and $-\mathrm{CH}_{2} \mathrm{Cl}$ groups. On the contrary, the Geotrichum candidum gives anti-Prelog halohydrins.

The excellent results and complementary enantioselectivities of the produced halohydrins obtained by using Rhodotorula glutinis CCT 2182 and Geotrichum candidum CCT 1205 in reduction of ethanones $\mathbf{1 a - j}$ are remarkable and highlight the potential of such approach to obtain separately the two isomers of the 1,2-aminoalcohols, by reaction of the easily obtainable epoxy with the appropriated amine (Scheme 2), as an alternative to the approach using the 
reduction of $\alpha$-azido-para-substituted acetophenones mediated by those microorganisms [28]. The separate synthesis of two enantiomers is important since the FDA Guidance for Development of New Stereoisomeric Drugs [44] says that "to evaluate the pharmacokinetics of a single enantiomer or mixture of enantiomers, manufacturers should develop quantitative assays for individual enantiomers in in vivo samples early in drug development." However, the products of biotransformation of $\mathbf{1 b}$-e and $\mathbf{1 g}$-j using Rhodotorula glutinis CCT 2182 may be used as important starting material for the preparation of the known pharmaceuticals products with $(R)$ configuration: Eliprodil from halohydrins $\mathbf{2} \mathbf{b}$ and $\mathbf{2 g}$; Tembamide from halohydrins $\mathbf{2 c}$ and $\mathbf{2 h}$; Aegeline from halohydrins $\mathbf{2 d}$ and $\mathbf{2} \mathbf{i}$; Nifenalol from halohydrins $2 \mathbf{e}$ and $\mathbf{2 j}$ (Figure 2).

\section{Conclusions}

The use of Rhodotorula glutinis CCT 2182 and Geotrichum candidum CCT 1205 in bioreduction reaction of 2-halo-1-(4substituted phenyl)-ethanones results in an important chiral halohydrins in high ee, excellent yield, and complementary enantioselectivity. These halohydrins may be used as intermediates in the synthesis of optically active substituted styrene oxides and aminoalcohols which have numerous industrial applications.

\section{Acknowledgments}

The authors thank FAPESP and CNPq for financial support.

\section{References}

[1] E. C. S. Brenelli, M. Carvalho, M. T. Okubo et al., "Enantioselective synthesis of $(R)-(-)$-1-phenylethanolamines using Baker's yeast reduction of some $\alpha$-substituted methyl phenyl ketones," Indian Journal of Chemistry B, vol. 31, pp. 821-823, 1992.

[2] C. Barbieri, L. Bossi, P. D’Arrigo, G. P. Fantoni, and S. Servi, "Bioreduction of aromatic ketones: preparation of chiral benzyl alcohols in both enantiomeric forms," Journal of Molecular Catalysis B, vol. 11, no. 4-6, pp. 415-421, 2001.

[3] R. N. Patel, "Biocatalysis: synthesis of chiral intermediates for drugs," Current Opinion in Drug Discovery and Development, vol. 9, no. 6, pp. 741-764, 2006.

[4] M. Breuer, K. Ditrich, T. Habicher et al., "Industrial methods for the production of optically active intermediates," Angewandte Chemie International Edition, vol. 43, no. 7, pp. 788$824,2004$.

[5] M. De Carvalho, M. T. Okamoto, P. J. S. Moran, and J. A. R. Rodrigues, "Baker's yeast reduction of $\alpha$-haloacetophenones," Tetrahedron, vol. 47, no. 12-13, pp. 2073-2080, 1991.

[6] A. Goswami, R. L. Bezbaruah, J. Goswami, N. Borthakur, D. Dey, and A. K. Hazarika, "Microbial reduction of $\omega$-bromoacetophe-nones in the presence of surfactants," Tetrahedron Asymmetry, vol. 11, no. 18, pp. 3701-3709, 2000.

[7] Y. Ni and J. H. Xu, "Asymmetric reduction of aryl ketones with a new isolate Rhodotorula sp. AS2.2241," Journal of Molecular Catalysis B, vol. 18, no. 4-6, pp. 233-241, 2002.
[8] L. C. Fardelone, J. A. R. Rodrigues, and P. J. S. Moran, "Bioreduction of alpha-haloacetophenones by Rhodotorula glutinis and Geotrichum candidum," Arkivoc, vol. 10, pp. 404410, 2003.

[9] L. H. Andrade, R. Polak, A. L. M. Porto, I. H. SchoenleinCrusius, and J. V. Comasseto, "Application of bioreduction by microorganisms in the enantioselective synthesis of alphasubstituted-1-phenylethanols," Letters in Organic Chemistry, vol. 3, no. 8, pp. 613-618, 2006.

[10] L. C. Rocha, H. V. Ferreira, E. F. Pimenta et al., "Biotransformation of $\alpha$-bromoacetophenones by the marine fungus Aspergillus sydowii," Marine Biotecnology, vol. 12, no. 5, pp. 552-557, 2010.

[11] A. E. P. M. Sorrilha, M. Marques, I. Joekes, P. J. S. Moran, and J. A. R. Rodrigues, "Reduction of phenylketones by immobilized Baker's yeast," Bioorganic and Medicinal Chemistry Letters, vol. 2, no. 2, pp. 191-196, 1992.

[12] M. Da Graça Nascimento, S. P. Zanotto, S. P. Melegari, and P. J. S. Moran, "Estudos de proteção da célula de Saccharomyces cerevisiae para utilização em reações de redução em meio orgânico," Química Nova, vol. 25, no. 4, pp. 567-571, 2002.

[13] Z. Ou, J. Wu, L. Yang, and P. Cen, "Asymmetric reduction of chloroacetophenones to produce chiral alcohols with microorganisms," Korean Journal of Chemical Engineering, vol. 25, no. 1, pp. 124-128, 2008.

[14] H. Lin, Y. Z. Chen, X. Y. Xu, S. W. Xia, and L. X. Wang, "Preparation of key intermediates of adrenergic receptor agonists: highly enantioselective production of $(R)$ - $\alpha$-halohydrins with Saccharomyces cerevisiae CGMCC 2.396," Journal of Molecular Catalysis B, vol. 57, no. 1-4, pp. 1-5, 2009.

[15] E. B. Kurbanoglu, M. Taskin, K. Zilbeyaz, and I. Hasenekoglu, "Efficient synthesis of (S)-1-(2-chlorophenyl)ethanol in the submerged culture of Alternaria alternata isolate," Chinese Journal of Catalysis, vol. 30, no. 4, pp. 370-374, 2009.

[16] L. C. Rocha, H. V. Ferreira, E. F. Pimenta et al., "Bioreduction of $\alpha$-chloroacetophenone by whole cells of marine fungi," Biotechnology Letters, vol. 31, no. 10, pp. 1559-1563, 2009.

[17] Y. Xie, J. H. Xu, and Y. Xu, "Isolation of a Bacillus strain producing ketone reductase with high substrate tolerance," Bioresource Technology, vol. 101, no. 3, pp. 1054-1059, 2010.

[18] F. R. Bisogno, E. Garcia-Urdiales, H. Valdes et al., "Ketonealcohol hydrogen-transfer equilibria: is the biooxidation of halohydrins blocked?" Chemistry, vol. 16, no. 36, pp. 11012$11019,2010$.

[19] J. A. R. Rodrigues, P. J. S. Moran, G. J. A. Conceição, and L. C. Fardelone, "Recent advances in the biocatalytic asymmetric reduction of acetophenones and $\alpha, \beta$-unsaturated carbonyl compounds," Food Technology and Biotechnology, vol. 42, no. 4, pp. 295-303, 2004.

[20] H. Hamada, T. Miura, H. Kumobayashi, T. Matsuda, T. Harada, and K. Nakamura, "Asymmetric synthesis of (R)-2chloro-1-(m-chlorophenyl)ethanol using acetone powder of Geotrichum candidum," Biotechnology Letters, vol. 23, no. 19, pp. 1603-1606, 2001.

[21] R. L. Hanson, S. Goldberg, A. Goswami, T. P. Tully, and R. N. Patel, "Purification and cloning of a ketoreductase used for the preparation of chiral alcohols," Advanced Synthesis and Catalysis, vol. 347, no. 7-8, pp. 1073-1080, 2005.

[22] W. Yang, J. H. Xu, Y. Xie, Y. Xu, G. Zhao, and G. Q. Lin, "Asymmetric reduction of ketones by employing Rhodotorula sp. AS2.2241 and synthesis of the $\beta$-blocker $(R)$-nifenalol," Tetrahedron Asymmetry, vol. 17, pp. 1769-1774, 2006.

[23] M. Chartrain, R. Greasham, J. Moore, P. Reider, D. Robinson, and B. Buckland, "Asymmetric bioreductions: application 
to the synthesis of pharmaceuticals," Journal of Molecular Catalysis B, vol. 11, no. 4-6, pp. 503-512, 2001.

[24] H. Antunes, L. C. Fardelone, J. A. R. Rodrigues, and P. J. S. Moran, "Chemoenzymatic syntheses of $(R)$-2-bromo-, $(R)-2$ chloro- and (R)-2-azido-1-(1,3-benzodioxol-5-yl)-1-ethanol," Tetrahedron Asymmetry, vol. 15, no. 17, pp. 2615-2620, 2004.

[25] H. Antunes, L. C. Fardelone, J. A. R. Rodrigues, and P. J. S. Moran, "Bioreduction of $\alpha$-substituted 3,4-(methylenedioxy)acetophenones by Rhodotorula glutinis and Geotrichum candidum," Brazilian Archives of Biology and Technology, vol. 49, pp. 133-138, 2006.

[26] N. Itoh, M. Matsuda, M. Mabuchi, T. Dairi, and J. Wang, "Chiral alcohol production by NADH-dependent phenylacetaldehyde reductase coupled with in situ regeneration of NADH," European Journal of Biochemistry, vol. 269, no. 9, pp. 2394-2402, 2002.

[27] Y. Yang, D. Zhu, T. J. Piegat, and L. Hua, "Enzymatic ketone reduction: mapping the substrate profile of a shortchain alcohol dehydrogenase (YMR226c) from Saccharomyces cerevisiae," Tetrahedron Asymmetry, vol. 18, no. 15, pp. 1799$1803,2007$.

[28] L. C. Fardelone, J. A. R. Rodrigues, and P. J. S. Moran, "Bioreduction of 2-azido-1-arylethanones mediated by Geotrichum candidum and Rhodotorula glutinis," Journal of Molecular Catalysis B, vol. 39, no. 1-4, pp. 9-12, 2006.

[29] D. P. Wyman and P. R. Kaufman, "The chlorination of active hydrogen compounds with sulfuryl chloride. I. Ketones," Journal of Organic Chemistry, vol. 29, no. 7, pp. 1956-1960, 1964.

[30] Fundação André Tosello Pesquisa e Tecnologia, Rua Latino Coelho 1301, 13087-010 Campinas-SP, Brazil, http://fat.org .br/.

[31] J. Hiratake, M. Inagaki, T. Nishioka, and J. Oda, "Irreversible and highly enantioselective acylation of 2-halo-1-arylethanols in organic solvents catalyzed by a lipase from Pseudomonas fluorescens," Journal of Organic Chemistry, vol. 53, no. 26, pp. 6130-6133, 1988.

[32] D. Basavaiah, G. J. Reddy, and V. Chandrashekar, "A novel and effective chiral phosphoramide catalyst for the boranemediated asymmetric reduction of prochiral $\alpha$-halo ketones," Tetrahedron Asymmetry, vol. 12, no. 4, pp. 685-689, 2001.

[33] D. Basavaiah, G. J. Reddy, and K. V. Rao, "Toward effective chiral catalysts containing the $\mathrm{N}-\mathrm{P}=\mathrm{O}$ structural framework for the borane-mediated asymmetric reduction of prochiral ketones," Tetrahedron Asymmetry, vol. 15, no. 12, pp. 18811888, 2004.

[34] S. Degni, C. E. Wilén, and A. Rosling, "Highly catalytic enantioselective reduction of aromatic ketones using chiral polymer-supported Corey, Bakshi, and Shibata catalysts," Tetrahedron Asymmetry, vol. 15, no. 9, pp. 1495-1499, 2004.

[35] D. Basavaiah, G. J. Reddy, and V. Chandrashekar, " $(2 S, 5 S)$ 1,3-Diaza-2-phospha-2-oxo-2-chloro-3-phenylbicyclo[3.3.0] octane: a novel chiral source for borane-mediated catalytic chiral reductions," Tetrahedron Asymmetry, vol. 13, no. 11, pp. 1125-1128, 2002.

[36] Z. L. Wei, Z. Y. Li, and G. Q. Lin, "anti-Prelog microbial reduction of aryl $\alpha$-halomethyl or $\alpha$-hydroxymethyl ketones with Geotrichum sp. 38," Tetrahedron, vol. 54, no. 43, pp. 13059-13072, 1998.

[37] D. D. Tanner, H. K. Singh, A. Kharrat, and A. R. Stein, "The mechanism of the reduction of $\alpha$-halo ketones by several models for NADH. Reduction by a SET-hydrogen atom abstraction chain reaction," Journal of Organic Chemistry, vol. 52, no. 11, pp. 2142-2146, 1987.
[38] D. D. Tanner and H. K. Singh, "Reduction of $\alpha$-halo ketones by organotin hydrides. An electron-transfer-hydrogen atom abstraction mechanism," Journal of Organic Chemistry, vol. 51, no. 26, pp. 5182-5186, 1986.

[39] D. D. Tanner and A. R. Stein, "On the mechanism of reduction by reduced nicotinamide adenine dinucleotide dependent alcohol dehydrogenase. $\alpha$-Halo ketones as mechanistic probes," Journal of Organic Chemistry, vol. 53, no. 8, pp. 16421646, 1988.

[40] D. D. Tanner and A. Kharrat, "Substrate-specific reduction mechanisms for NADH models. Reduction of N-methylacridinium iodide and $\alpha, \alpha, \alpha$-trifluoroacetophenone," Journal of Organic Chemistry, vol. 53, no. 8, pp. 1646-1650, 1988.

[41] J. M. Smolen, E. J. Weber, and P. G. Tratnyek, "Molecular probe techniques for the identification of reductants in sediments: evidence for reduction of 2-chloroacetophenone by hydride transfer," Environmental Science and Technology, vol. 33, no. 3, pp. 440-445, 1999.

[42] L. M. Aleixo, M. De Carvalho, P. J. S. Moran, and J. A. R. Rodrigues, "Hydride transfer versus electron transfer in the baker's yeast reduction of $\alpha$-haloacetophenones," Bioorganic and Medicinal Chemistry Letters, vol. 3, no. 8, pp. 1637-1642, 1993.

[43] V. Prelog, "Specification of the stereospecificity of some oxidoreductases by diamond lattice sections," Pure and Applied Chemistry, vol. 9, no. 1, pp. 119-130, 1964.

[44] http://www.fda.gov/Drugs/GuidanceComplianceRegulatoryInformation/Guidances/ucm122883.htm, December 2010. 

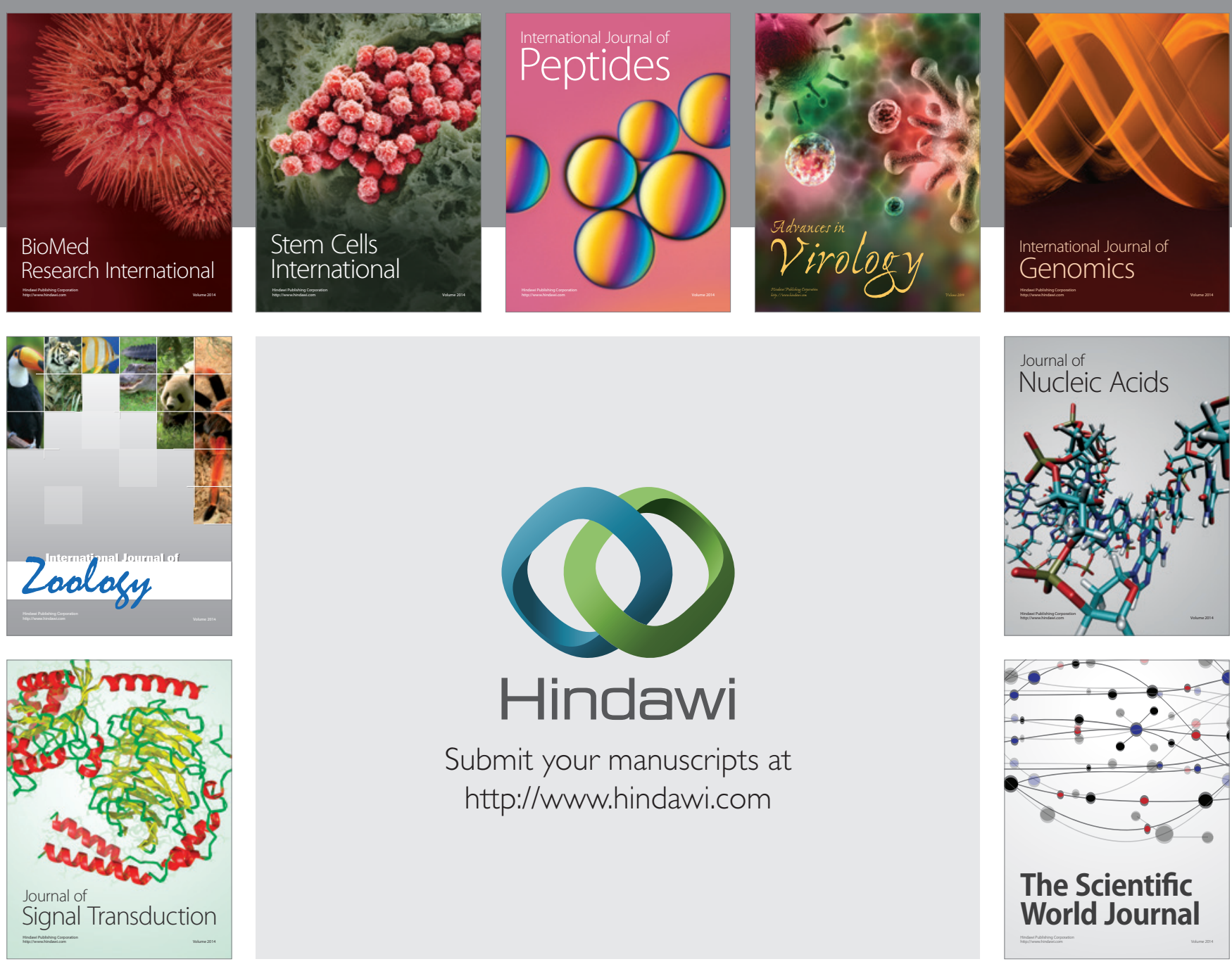

Submit your manuscripts at

http://www.hindawi.com
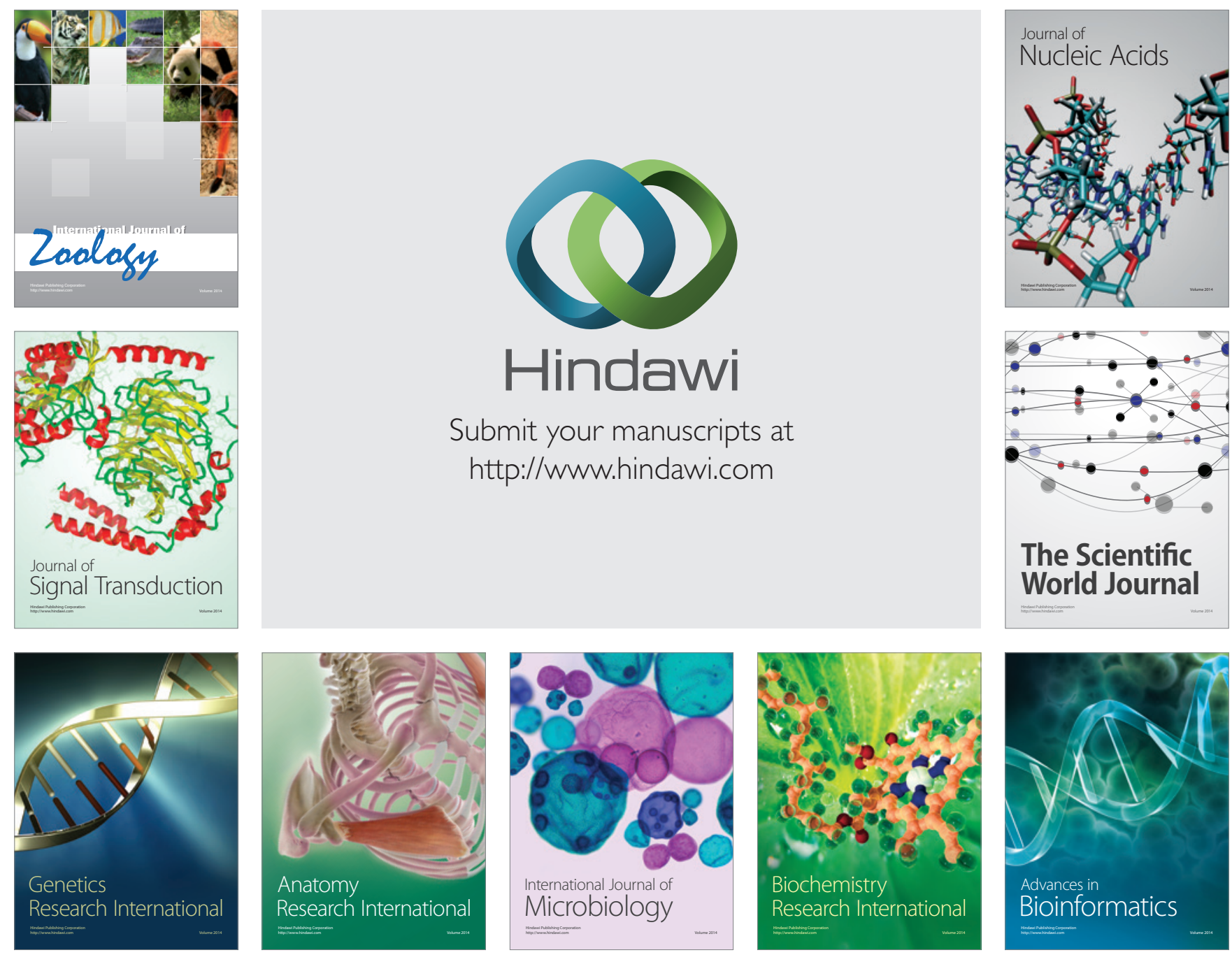

The Scientific World Journal
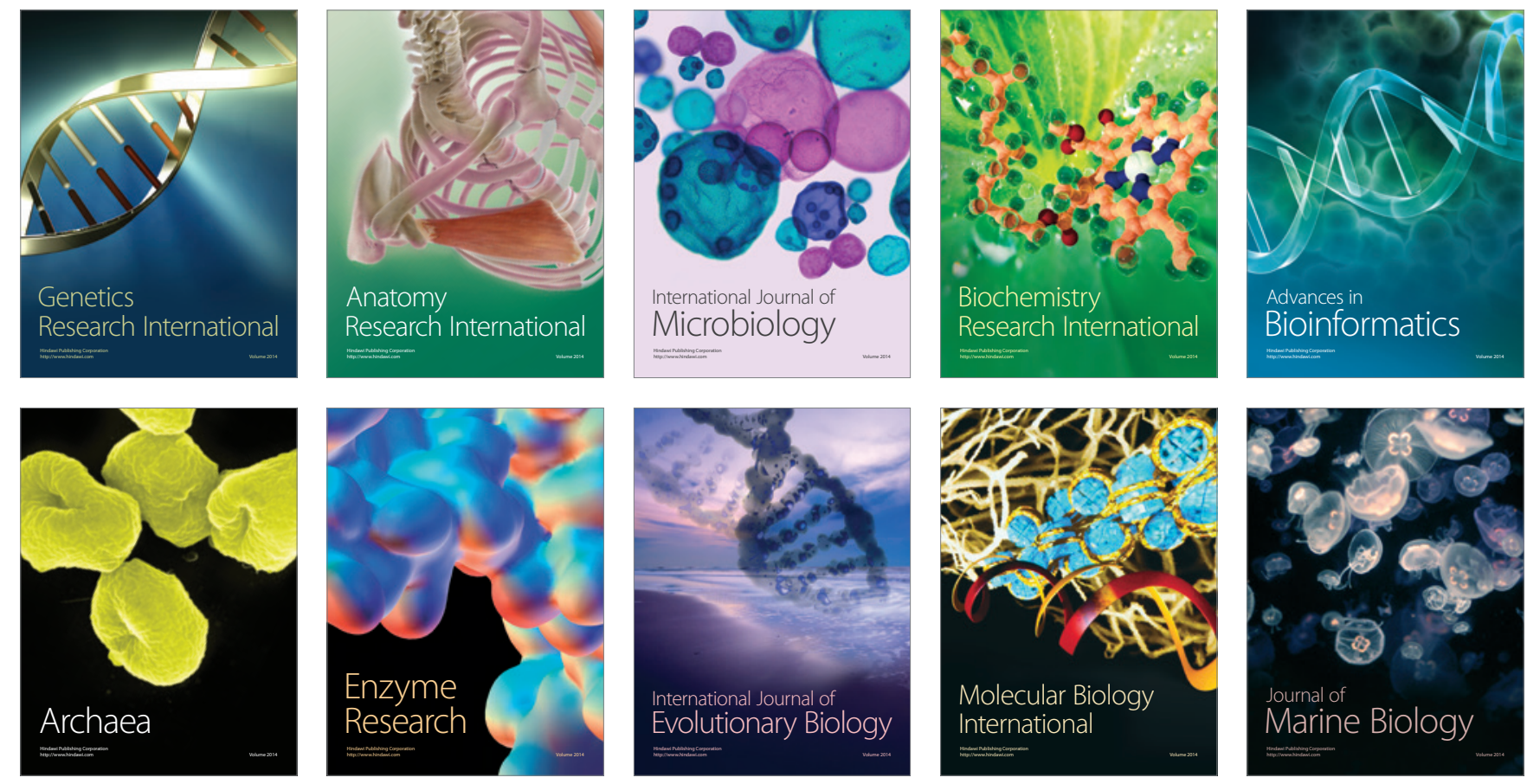